\title{
Die scheinheiligen Jugendschützer und ihre Helfer
}

\section{Rainer M. Kaelin}

Dr. med., Facharzt für Pneumologie und Innere Medizin, vormals Vizepräsident der Lungenliga Schweiz und der Lungenliga Waadt, Vizepräsident von OxyRomandie/OxySuisse; Mitglied der FMH

\section{Zigarettenhersteller versuchen Öffentlichkeit und Parlamentarier von ihrem un- wahrscheinlichen Wandel zu überzeugen: Von nun an wollen sie nur noch, dass erwachsene Raucher auf E-Zigaretten und alternative Produkte umsteigen.}

Als der Arzt und ehemalige WHO-Angestellte, Dr. Derek Yach, als eben designierter Präsident der Philip Morris (PM) Stiftung "For a smokefree world» 2017 verkündete, PM wolle, dass «das Rauchen in dieser Generation ende», klang das nicht glaubwürdig. Die Verlagsbeilage der NZZ vom 22. Mai mit dem Titel «Es gibt kein zurück mehr. Philip Morris will die Zigarettenproduktion einstellen» generierte ebenfalls Skepsis. Das neueste Element dieser offensichtlichen PR-Aktionen fand man kürzlich im Interview mit dem Chef von British American Tobacco (BAT) «Niemand ist mehr besonders stolz darauf zu rauchen" [1]. Der nichtrauchende CEO Mads Larsen ist selbstverständlich für den Jugendschutz und «möchte nicht, dass (seine) zwölfjährige Tochter nach Hause kommt und sagt, sie habe angefangen zu dampfen». Eltern werden ihm beipflichten, wenn er zu den Marketingmethoden der E-Zigarette Juul bemerkt, "was in den USA passiert, ist schockierend! Wir müssen schauen, dass die Schweiz von solchen Entwicklungen

Der nichtrauchende CEO Mads Larsen ist selbstverständlich für den Jugendschutz.

verschont bleibt.» Larsen erklärt, ähnlich wie Jonathan Green von Juul labs [2]: «Ich lege die Hand dafür ins Feuer, dass wir unsere Produkte nur an Leute $a b$ 18 Jahre verkaufen, also an erwachsene mündige Raucher. So sieht es unsere Selbstregulierung vor, die seit 2005 besteht.»

\section{Widersprüchliche Aussagen}

Dass BAT wie auch Juul und PM die neueren Produkte nur an erwachsene Raucher/innen verkaufen will, kann nicht ehrlich gemeint sein. Denn ohne neu- rekrutierte Nikotinsüchtige würde dieses Geschäftsverhalten zur Folge haben, dass der Markt in einer Generation austrocknet. Wenn das E-Zigaretten-Angebot abhängige Raucher ansprechen soll, dann hängt das Bestehen dieses Geschäftes davon $a b$, dass nikotinabhängige Raucher generiert werden, und dies geschieht mit dem An-

fixen von Jungen. Und tatsächlich will Larsen «Marktanteile von unseren Konkurrenten gewinnen. Das ist ohne Werbung nicht möglich.» Sogenannte «Selbstregulierung» der Werbung, die den Tabakkonsum von Jugendlichen verhindern soll, ist eine Farce: Swiss Cigarette (d.h. PM, BAT und Japan Tobacco International, JTI) vereinbarten 2005 mit der vom Werbeverband getragenen Lauterkeitskommission (LK), dass diese die Verstösse gegen den Ehrencodex beurteilen soll. Im Gegensatz zu Beanstandungen in anderen Bereichen sieht jedoch das LK-Reglement vor, Übertretungen der Tabakindustrie nicht zu sanktionieren, sondern als «Empfehlungen» weiterzuleiten an ... Swiss Cigarette [3]! Kein Wunder, dass Larsen die Volksinitiative «Ja zum Schutz der Kinder und Jugendlichen vor Tabakwerbung» ablehnt. Denn: «Fast die Hälfte der Werbeausgaben fliesst bei BAT aktuell in die neuen Produkte. Ausserdem: Was ist mit Alkohol, Fett und Zucker, die ebenfalls die Gesundheit belasten können? Konsequenterweise müsste man Werbung für solche Produkte ebenfalls verbieten.» Diese typische 
Sprachregelung von Gewerbeverband, Werbe- und Getränkeindustrie sowie der SVP [4] ist seit dem letzten Jahrhundert üblich. Ein internes Papier von R. J. Reynolds (später JTI) hält an dieser PR-Argumentation fest, da sie erfolgreich zu vernebeln half, dass die Gefährdung durch Tabak auf dem hohen Suchtpotential des Nikotins für Junge beruht [5]. Die schwankenden Gefühle zwischen CEO und dem verantwortungsbewussten Bürger Larsen treten zutage, als er die Behauptung der Tabakbranche kommentiert, Zigaretten seien

\section{Dass offizielle Angaben die Raucherquoten der Schweiz seit Jahren grob unterschätzen, wird nicht angesprochen.}

ein Genussmittel neben vielen anderen: «Nikotin hat ein hohes Suchtpotential, deshalb ist ein massvoller Genuss - anders als beim Rotwein - nicht immer ganz einfach [...] Schon täglich 5 Zigaretten zu konsumieren ist mittel- bis langfristig sicher nicht unproblematisch.» Doch stimmt er in den Refrain ein: "Der Staat kann auf das Konsumverhalten [...]nur begrenzt Einfluss nehmen. In Frankreich darf unsere Branche überhaupt keine Werbung schalten [...] und doch rauchen dort viel mehr Menschen als in der Schweiz.» Dass offizielle Angaben die Raucherquoten der Schweiz seit Jahren grob unterschätzen [6], wird nicht angesprochen.

\section{Die liberale Schweizer Tabak- gesetzgebung}

In derselben NZZ-Ausgabe findet sich der Artikel «Die relative liberale Schweizer Tabakgesetzgebung wird verschärft. Sieben Antworten zum Umbruch auf dem Tabakmarkt». Die Autoren erwähnen das Rauchverbot in Bahnhöfen, die Initiative "Ja zum Schutz der Kinder und Jugendlichen vor Tabakwerbung" und die debattierten Regelungen des Tabakproduktegesetzes: das Verkaufsverbot an Minderjährige, die Testkäufe, um das Verbot umzusetzen und dass Werbung, die sich speziell an Minderjährige richtet, nicht mehr erlaubt sein soll. Was theoretisch schon jetzt der Fall wäre, wenn die «Selbstregelung» durch die Lauterkeitskommission greifen würde. Für E-Zigaretten und Snus wird von "differenzierten Regeln" gesprochen und dass Werbung, Marketing und Sponsoring dieser Produkte wie für herkömmliche Tabakprodukte geregelt werden sollen, d.h. beinahe gar nicht, da nur konventionelle Werbung betroffen ist, nicht aber die modernen Methoden der Produktepromotion. Der Forderung der Tabakindustrie nach Ausnahmen scheint entsprochen worden zu sein, denn alternative Produkte werden in einer «eigenen Kategorie gere- gelt», müssen einen allgemeinen Warnhinweis tragen, jedoch ohne das abschreckende Bild der Zigarettenpackungen. Der sachliche, aber unkritische Artikel unterlässt es, die unterschätzte offizielle Raucherquote der Schweiz [6] und die Tabakrahmenkonvention der WHO (FCTC) zu kommentieren, die seit 2004, ihrer parlamentarischen Ratifizierung harrt. Die FCTC gebietet, dass Werbung, Marketing und Sponsoring für Tabakund Nikotinprodukte "umfassend» verboten werden und dass die Präventionsmassnahmen vor der Unterwanderung durch die Industrie zu schützen seien. Der NZZ-Leser erfährt nicht, dass ausgerechnet der freisinnige Ständerat Dittli aus Uri an der Sitzung der Gesundheitskommission vom 19. Februar forderte, das TabPG solle durch Werbeverbote verbessert werden, um die Ratifizierung der FCTC zu ermöglichen [7]. Im Jahre 2016 hatte derselbe Ständerat die Rückweisung des Entwurfes and den Bundesrat ausgelöst mit der Aussage, er kenne keine Daten, die belegen, dass Werbeverbote sinkende Raucherquoten zur Folge hätten!

\section{Der unlösbare Konflikt}

Dass die Tabakindustrie Parlamentarier und öffentliche Meinung mit allen Mitteln zu überzeugen versucht, Nikotin sei banal und nur der verbrannte Tabak sei für das öffentliche Gesundheitsproblem der Tabakepidemie verantwortlich, ist nicht erstaunlich. Denn die Anerkennung der Tatsache, dass die in der Jugend verankerte Nikotinabhängigkeit die Tabakepidemie mit ihren Folgen verursacht, stellt ihre Geschäftsgrundlage grundlegend in Frage und macht sie zum unversöhnlichen Gegner der öffentlichen Gesundheit. Diese Tatsache ist aber auch die Grundlage der auf wissenschaftlicher Evidenz basierenden Forderung der FCTC, dass Werbe-, Promotions- und Sponsoringverbote umfassend sein müssen, um wirksamen Jugendschutz zu garantieren. Die irreführende Argumentation «Schadensminderung» durch weniger schädliche Produkte lag schon dem Marketing der Filter- und der

\section{Dass die Tabakindustrie mit allen Mitteln} zu überzeugen versucht, Nikotin sei banal, ist nicht erstaunlich.

«milden» Zigarette zugrunde, als man begann, Nikotin und die karzinogenen Komponenten des Rauches zu messen. Wobei die Tabakindustrie (analog wie später die Autoindustrie) Öffentlichkeit und Behörden in die Irre führte. Von diesen klassischen Produkten wurde nie eine geringere Schädlichkeit nachgewiesen, sie kurbelten aber den Tabakkonsum in der jungen und weiblichen Bevölkerung wesentlich an. Content Mar- 
keting zum Thema «Schadensminderung» zur Banalisierung von Nikotin und Nikotinsucht findet sich seit der E-Zigarette wieder vermehrt in redaktionellen und bezahlten Medienbeiträgen. Die dabei kaum hinterfragten Botschaften sind die «95\% geringere Schädlichkeit der E-Zigarette» (Prof. Etter 2013), wobei Schädlichkeit und Schadstoffgehalt vermengt werden, «Werbung ist Information des mündigen Kunden», «Ein legales Produkt soll auch legal beworben werden dürfen» (Ständerat Filippo Lombardi, Präsident von Kommunikation Schweiz, 2016), «Kurswechsel in der Schweizer Tabakpolitik» (Fachverband Sucht 2017), «Sinnvolle Alternativen zu Tabakzigaretten» (Prof. M. Kunze 2017).

\section{Vergiftung von Meinungsbildung und demokratischem Prozess}

Im vergangenen Jahrhundert hat die multinationale Tabakindustrie auf Kosten der Gesundheit ihrer Kunden sowie des Gesundheitssystems enorme Gewinne eingefahren, weil sie die Nikotinaufnahme aus ihren Produkten ins Gehirn von Jugendlichen mit wissen-

\section{Die Tabakindustrie hat auf Kosten der Gesund-} heit ihrer Kunden sowie des Gesundheitssystems enorme Gewinne eingefahren.

schaftlichen Methoden verbesserte. Gleichzeitig hat sie systematisch durch Werbe-, Promotions- und Sponsoringmassnahmen und kartellistische Einflussnahme

Korrespondenz: Dr. Rainer M. Kaelin Plantay 53

CH-1163 Etoy VD palmier.kaelin[at] bluewin.ch auf breiter Ebene ihre Manipulationen und deren Folgen in Gesellschaft und vor Behörden erfolgreich verheimlicht. Ihre Machenschaften (und nicht die Blätter der Tabakpflanze) sind für das öffentliche Gesund- heitsproblem der Tabakepidemie verantwortlich. Parlamentarier und Medienschaffende wären gut beraten, aus dem fundamentalen Interessenkonflikt zwischen Tabak-/

Nikotinindustrie und öffentlicher Gesundheit kritische Schlüsse zu ziehen, wenn sie über Jugendschutz im Tabakproduktegesetz, die Tabakrahmenkonvention der WHO und über die Volksinitiative «Kinder ohne Tabak» zu entscheiden und zu berichten haben.

\section{Literatur}

1 NZZ vom 1.6.2019: «Niemand ist mehr besonders stolz darauf zu rauchen". Interview mit Mads Larsen, von Erich Aschwanden und Simon Hehli.

2 Tages-Anzeiger vom 5.12.2018: «Ich bin selber Vater von zwei Kindern".

3 R. M. Kaelin: Jugendschutz ohne Tabakwerbeverbot? Schweiz Ärzteztg. 2017;98(41):1347-9.

4 Bundesgesetz über Tabakprodukte (TabPG). Vernehmlassungsantwort der Schweizerischen Volkspartei (SVP). 9.9.2014

5 R. J. Reynolds Tobacco International: Swiss Advertising Ban Case Study. 1993 November 28. RJ Reynolds Records (Author unknown). Internet: https://www.industrydocuments.ucsf.edu/docs/ pzmh0077 (accessed 22.5.2019).

6 J. Jacob, J. Cornuz, P. Diethelm. Prevalence of Tobacco smoking in Switzerland: do reported numbers underestimate reality? Swiss Medical Weekly. 2017;147:w14437.

7 Lorenz Hongegger. Lukratives Mandat: Der «Briefträger der Tabaklobby» wechselt die Seite. Schweiz am Wochenende, 2.3.2019.

Bildnachweis

(c) Annepunch | Dreamstime.com 